\title{
A double-blind, randomized, multicenter phase 2 study of prasugrel versus placebo in adult patients with sickle cell disease
}

Ted Wun ${ }^{1 *}$, Denis Soulieres ${ }^{2}$, Andrew L Frelinger ${ }^{3}$, Lakshmanan Krishnamurti ${ }^{4}$, Enrico M Novelli ${ }^{5}$, Abdullah Kutlar ${ }^{6}$, Kenneth I Ataga ${ }^{7}$, Charles L Knupp ${ }^{8}$, Lillian E McMahon ${ }^{9}$, John J Strouse ${ }^{10}$, Chunmei Zhou ${ }^{11}$, Lori E Heath ${ }^{11}$, Chuke E Nwachuku ${ }^{12}$, Joseph A Jakubowski ${ }^{11}$, Jeffrey S Riesmeyer ${ }^{11}$ and Kenneth J Winters ${ }^{11}$

\begin{abstract}
Background: Platelet activation has been implicated in the pathogenesis of sickle cell disease (SCD) suggesting antiplatelet agents may be therapeutic. To evaluate the safety of prasugrel, a thienopyridine antiplatelet agent, in adult patients with SCD, we conducted a double-blind, randomized, placebo-controlled study.

Methods: The primary endpoint, safety, was measured by hemorrhagic events requiring medical intervention. Patients were randomized to prasugrel $5 \mathrm{mg}$ daily $(n=41)$ or placebo $(n=21)$ for 30 days. Platelet function by VerifyNow ${ }^{\circledR}$ P2Y12 and vasodilator-stimulated phosphoprotein assays at days 10 and 30 were significantly inhibited in prasugrel- compared with placebo-treated SCD patients.

Results: There were no hemorrhagic events requiring medical intervention in either study arm. Mean pain rate (percentage of days with pain) and intensity in the prasugrel arm were decreased compared with placebo. However, these decreases did not reach statistical significance. Platelet surface P-selectin and plasma soluble P-selectin, biomarkers of in vivo platelet activation, were significantly reduced in SCD patients receiving prasugrel compared with placebo. In sum, prasugrel was well tolerated and not associated with serious hemorrhagic events.
\end{abstract}

Conclusions: Despite the small size and short duration of this study, there was a decrease in platelet activation biomarkers and a trend toward decreased pain.

Keywords: Prasugrel, Sickle cell disease, Thienopyridine, Platelet function

\section{Background}

Sickle cell disease (SCD) (the common term used for the clinical syndrome that results from homozygous hemoglobin $\mathrm{S}$; compound heterozygous $\mathrm{Hb} \mathrm{S/C}$; and compound heterozygous $\mathrm{Hb} \mathrm{S} / \beta^{0 /+}$-thalassemia) results from a mutation in the $\beta$-globin gene. The clinical manifestations of disease are due to hemolysis, and intermittent microvascular occlusion marked by painful vaso-occlusive crisis (VOC) and eventual end-organ damage from repeated bouts of ischemiareperfusion injury resulting in significant disabilities and early mortality. SCD pathophysiology is multi-factorial. The reduced intracellular solubility of $\mathrm{HbS}$ leads to intra-

\footnotetext{
* Correspondence: twun@ucdavis.edu

'University of California, Davis Cancer Center, 4501 X St., Ste. 3016, Sacramento, CA 95817, USA

Full list of author information is available at the end of the article
}

erythrocytic hemoglobin polymerization and loss of red cell deformability under conditions of hypoxia and acidosis [1]. The sickle red blood cell is also abnormally adhesive to activated endothelial cells even in the absence of sickling [2]. Neutrophils and monocytes are activated and contribute to vascular occlusion [3-6]. Numerous studies have also shown activation of the hemostatic system, including the coagulation cascade and platelets [7-9]. Activation of all these cells and the coagulation system likely contributes to a prothrombotic state producing the acute and chronic disease manifestations.

Previous work, using various soluble and immunological biomarkers, has shown that platelets are activated in patients with SCD [10-12]. Some data have suggested increased platelet activation during VOC [12-14]. Possible mechanisms whereby activated platelets contribute to the pathogenesis of VOC include release of thrombospondin 1

\section{Ciomed Central}


during platelet activation that supports aberrant red blood cell (RBC) adhesion to endothelium and matrix proteins, and platelets directly mediating $\mathrm{RBC}$ adherence to endothelial cells and capture onto neutrophils and monocytes (heterotypic cell-cell adherence; and activation of neutrophils and monocytes via formation of platelet-monocyte and platelet-neutrophil aggregates). The activated leukocytes augment the overall inflammatory state and promote further vascular and tissue damage.

There have been studies of antiplatelet agents for SCD patients including use of aspirin [15] and the first generation thienopyridine adenosine diphosphate (ADP) receptor inhibitor, ticlopidine [16,17]. In general, these studies have been relatively small, underpowered, and without control groups. Prasugrel, a third-generation platelet P2Y12 ADP receptor antagonist, is FDAapproved in combination with aspirin for treatment of patients with acute coronary syndrome undergoing percutaneous coronary intervention. We performed a phase 2, double-blind, randomized trial of prasugrel compared with placebo in patients with SCD. The primary safety outcome was treatment-emergent hemorrhagic events requiring medical intervention. Secondary safety outcomes included all adverse events (AEs) including those that required study drug discontinuation. The efficacy outcome measures were frequency and intensity of pain ascertained by self-administered pain diary; frequency of pain requiring medical attention; pharmacodynamic effects on platelets measured by VerifyNow ${ }^{\circledR}$ P2Y12 reaction units (PRU) and vasodilator-associated stimulated phosphoprotein (VASP) platelet reactivity index (PRI); and biomarkers of platelet activation. We hypothesized that prasugrel would: 1) be well tolerated, 2) inhibit platelet activation, and 3) decrease pain.

\section{Methods}

This was a double-blind, randomized, multicenter trial to assess the safety of prasugrel $5 \mathrm{mg}$ PO (by mouth) daily compared with placebo in adult patients with SCD (clinicaltrials.gov \#NCT01167023). The study was conducted at 18 sites in the United States and Canada from 26 August 2010 to 13 June 2011. The study was approved by the local institutional review boards and was performed in compliance with principles of good clinical practice (GCP) and in accordance with the provisions of the Declaration of Helsinki. Each patient voluntarily signed an informed consent document before entering the study.

\section{Eligibility}

To be included in the study, patients had to be adults 18 to 55 years of age with SCD (genotypes $\mathrm{HbSS}, \mathrm{HbSC}$, $\mathrm{HbS}-\beta^{0}$-thalassemia and $\mathrm{HbS}-\beta^{+}$-thalassemia), who did not have a diagnosis of acute VOC within 30 days of the study screening visit. Patients with severe hepatic or renal dysfunction, hematocrit $<18 \%$, and those at risk of excessive bleeding complications including platelet count $<100,000$ per cubic millimeter, prior history of bleeding disorders, hemorrhagic or ischemic stroke, retinal hemorrhage, a transient ischemic attack (TIA), or intracranial hemorrhage were excluded from the study. We felt these conservative exclusion criteria were warranted given this new patient population. Use of aspirin or other antithrombotics was not allowed within 10 days of entry or during the study. Non-steroidal antiinflammatory drugs (NSAIDs) for treatment of pain were not permitted in the 5 days prior to randomization or for $\geq 5$ consecutive days during the study period. Use of hydroxyurea was permitted in patients already on a stable dose 30 days prior to randomization.

\section{Study design}

The study consisted of 2 phases (Phase A and Phase B) (Figure 1) and employed an adaptive design with decisions about dose allocations made as the trial progressed. During each phase, patients were randomized to prasugrel or placebo in a 2:1 manner and stratified by sickle-cell genotype $\left(\mathrm{HbSS}, \mathrm{HbS}-\beta^{+}\right.$-thalassemia and $\mathrm{HbS}-\beta^{0}$-thalassemia genotype patients in one stratum, and $\mathrm{HbSC}$ genotype patients in another stratum). We stratified in this way because some data [18,19], though not all $[10,14]$, suggest more pronounced platelet activation in patients with HbSS than HbSC. In addition, our limited studies showed no difference in platelet activation between $\mathrm{Hb} \mathrm{S}-\beta^{+}$-thalassemia and HbSS patients [10]. Patients underwent screening (Visit 0 ) and returned to the site for Visit 1 (0 to 10 days after screening), Visit $2(10 \pm 2$ days after Visit 1$)$, and Visit 3 (30 \pm 3 days after Visit 1$)$. Patients were contacted by phone for Visit 4 (30 days after last dose of study drug) to collect information on adverse events (AEs) and serious adverse events (SAEs).

All patients randomized to prasugrel during Phase A received a 5-mg/day dose. When pharmacodynamic (PD) data from Visit 2 were available for $\geq 16$ patients treated with prasugrel, a Data Monitoring Committee (DMC) assessed the results and reviewed initial safety data prior to making a determination regarding the Phase B prasugrel dose. If at Visit $2, \geq 60 \%$ of $\geq 16$ patients treated with prasugrel in Phase $A$ had the equivalent of $<25 \%$ inhibition of platelet aggregation (IPA) as measured by light transmission aggregometry (LTA), then a 7.5-mg/day prasugrel dose was to be used in Phase B for patients who weighed $\geq 60 \mathrm{~kg}$, and a 5 -mg/day prasugrel dose would be used in patients who weighed $<60 \mathrm{~kg}$. Otherwise, Phase $\mathrm{B}$ was to use a 5 -mg/day prasugrel dose for all patients, regardless of weight. After the DMC determined the Phase B dose, future patients were randomized into Phase $\mathrm{B}$, while all 


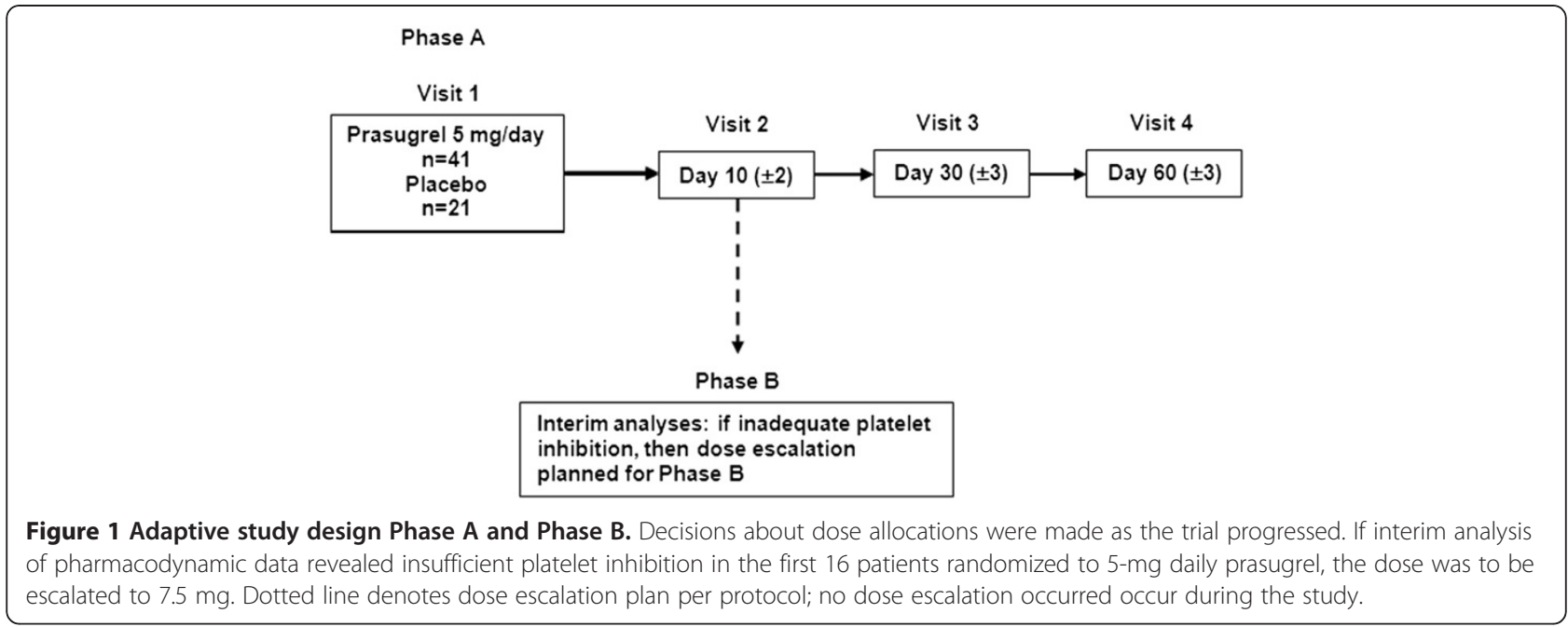

patients randomized in Phase A completed their scheduled follow-up. Based on the accumulated data, as reviewed by the DMC, a 5-mg daily dose was used for the remainder of the study. The duration of active treatment and pain assessment was 30 days and the total duration with follow-up was 60 days.

\section{Endpoint assessment}

The primary endpoint was the incidence of hemorrhagic events requiring medical attention: an unscheduled visit or call to a medical provider due to a complaint of bleeding while on study drug. Bleeding events that did not require medical attention were captured, in retrospect, as adverse events at routine assessments. This endpoint was chosen because sickle cell patients represented a new population for this drug and it was felt necessary to have robust safety data in adults prior to proceeding with a planned study in pediatric patients. Endpoint ascertainment was achieved by interviewing patients at each visit. Additional safety evaluations included the assessment of clinical laboratory test results, vital sign measurements, 12-lead electrocardiogram results, fundoscopies, and AEs. Information on SAEs was collected at occurrence as well as by telephone interview 30 days after the last dose of study drug. Diseaserelated treatment emergent adverse effects (TEAEs) including VOC, acute chest syndrome, hepatic sequestration, and stroke were reported as AEs or SAEs as appropriate.

Efficacy was a secondary endpoint as assessed by incidence of any pain events requiring medical attention in the study. In addition, the efficacy of prasugrel $(5 \mathrm{mg})$ compared with placebo was measured by monitoring the frequency and intensity of pain related to SCD as recorded in patient pain diaries each day for 30 days. A scale of 0 to 9 was used to evaluate pain intensity, with 0 indicating no pain, and 9 indicating unbearable pain.

\section{Pharmacodynamic and biomarker evaluations}

Platelet inhibition was assessed by the Accumetrics VerifyNow ${ }^{\circledR}$ P2Y12 assay and the VASP phosphorylation assay as previously described [20,21]. Assay data was reported as P2Y12 reaction units (PRU) and percent inhibition (device reported) for the VerifyNow ${ }^{\circledR}$ P2Y12 assay and platelet reactivity index (PRI) for VASP.

Platelet activation was assessed by immunoassay determination of plasma, serum, and cellular biomarkers of platelet activation. Multi-color fluorescent activated cell sorting (FACS) and monoclonal antibodies were used to determine platelet $\mathrm{P}$-selectin expression using previously published protocols [22,23]. Soluble P-selectin (sPselectin), soluble $\mathrm{CD} 40$ ligand (sCD40L), thromboxane B2 (serum TXB2), and prothrombin fragment F1.2 (F1.2) were determined using standard enzyme-linked immunoassays according to manufacturer's directions.

Pharmacodynamic parameters and biomarkers were assessed at Visits 1, 2, and 3. VerifyNow ${ }^{\circledR}$ P2Y12 was performed locally; platelet surface P-selectin was determined at the Center for Platelet Research Studies; and all other evaluations were performed at a central laboratory. Approximately $30-50 \mathrm{~mL}$ of venous blood was collected by venipuncture at each visit for use in PD and biomarker testing.

\section{Statistical analysis}

Patient characteristics were summarized by treatment group with values presented as mean \pm standard deviation (SD) for continuous variables, and counts (percentages) for categorical variables. An analysis of variance (ANOVA) model for continuous variables and a Fisher's exact test for the categorical variables were used for comparisons between treatment groups. The number and percentage of patients experiencing safety endpoints including the frequency of hemorrhagic events requiring 
medical intervention were summarized by treatment group.

Pain rate and pain intensity were summarized by treatment group using descriptive statistics. Pain rate was defined as the number of days reported with any pain related to SCD divided by the number of daily pain diaries completed. Pain intensity was the sum of a patient's pain scores on a scale of 0 to 9 divided by the number of daily pain diaries completed. An analysis of covariance (ANCOVA) model with baseline pain intensity, treatment group, and sickle-cell genotype (HbSS, HbS- $\beta^{0}$ thalassemia and $\mathrm{HbS}-\beta^{+}$-thalassemia in one stratum and $\mathrm{HbSC}$ in another stratum) in the model was used for treatment group comparison. A sensitivity analysis using only data from patients who completed 20 or more daily pain diaries was performed to verify that missing data did not introduce bias. The number of patients experiencing a pain event that required medical attention was compared between treatment groups using Fisher's exact test.

Pharmacodynamic and biomarker results were summarized by treatment group and visit. The post-baseline results for each PD and biomarker parameter were analyzed using a mixed-effects model repeated measures (MMRM) analysis with the fixed effects of treatment, baseline parameter result, sickle-cell genotype, visit, and visit-by-treatment interaction, as well as a patient random effect included in the model. A compound symmetry variance-covariance structure was used to estimate within-patient errors.

Because a more conventional way to stratify analysis for sickle cell genotypes is to compare HbSS and HbS$\beta^{0}$-thalassemia with $\mathrm{HbSC}$ and $\mathrm{HbS}-\beta^{+}$-thalassemia (based on disease severity), the ANCOVA analysis for pain rate and pain intensity and the MMRM analysis for PD and biomarker parameters were repeated with adjustment of genotype based on the conventional stratification. This sensitivity analysis did not change any of the results for statistical significance on the treatment comparison.

The sample size calculation for this study is limited due to the rarity of the primary endpoint of hemorrhagic events requiring medical intervention, and so is supported by a power calculation for the efficacy endpoints. A sample size of 60 patients with the ratio of prasugrel to placebo as 2:1 provides $80 \%$ power to detect approximately $60 \%$ reduction in the mean pain rate at the 2 -sided 0.05 level. This results in the ability to detect an effect size of 0.78 .

\section{Results}

A total of 62 patients were randomly assigned to treatment (prasugrel $[n=41]$, placebo $[n=21]$ ) and were included in the Intent-to-Treat (ITT) analysis set (Figure 2). A total of 57 patients completed the study (39 [95.1\%] prasugrel; 18 [85.7\%] placebo). No patients received aspirin, nor NSAIDs, during the study. One patient was found not to have SCD ( $\beta$-thalassemia trait only) and another patient was enrolled and randomized as the study ended and it was decided not to proceed with treatment of that patient.

The baseline characteristics of the treatment groups were well balanced (Table 1). The mean pain intensity for all patients was low, likely reflecting that these patients were clinically stable without recent painful episodes. A greater proportion of patients randomized to prasugrel had a history of acute chest syndrome and systemic and pulmonary hypertension.

No patient experienced hemorrhagic adverse events that required acute medical intervention (Table 2), the primary endpoint of the study. Eight patients in the prasugrel arm experienced 9 hemorrhagic AEs, the majority of which were mild and possibly related to the study drug (Table 3) but did not result in study drug

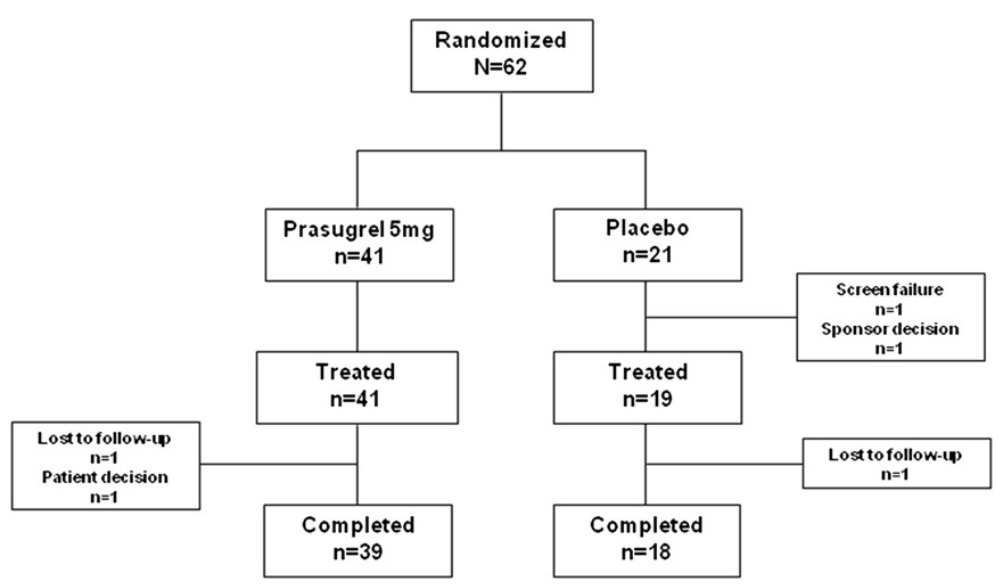

Figure 2 Patient distribution. A total of 62 patients were randomly assigned to treatment (prasugrel [41], placebo [21]) and were included in the Intent-to-Treat (ITT) analysis set. 
Table 1 Baseline characteristics and medical history

\begin{tabular}{|c|c|c|c|}
\hline & Prasugrel $5 \mathrm{mg} \mathrm{n}=41^{*}$ & Placebo $n=21$ & $\mathrm{p}$ value \\
\hline Age (mean, years) & 32.9 & 31.5 & 0.553 \\
\hline Female, n (\%) & $21(51.2)$ & $9(42.9)$ & 0.598 \\
\hline Body Weight (mean, kg) & 78.3 & 68.6 & 0.021 \\
\hline Hydroxyurea & 18 & 9 & \\
\hline Sickle-cell Genotype, n (\%) & & & $>0.999$ \\
\hline $\mathrm{Hb} \mathrm{S} / \beta 0$ thalassemia & $2(5.0)$ & $1(4.8)$ & \\
\hline $\mathrm{Hb} \mathrm{S} / \beta \tilde{+}$ thalassemia & $4(10.0)$ & $2(9.5)$ & \\
\hline $\mathrm{Hb}$ SC & $10(25.0)$ & $5(23.8)$ & \\
\hline $\mathrm{Hb}$ SS & $24(60.0)$ & $13(61.9)$ & \\
\hline Pain Intensity & & & 0.473 \\
\hline Mean & 1.8 & 2.4 & \\
\hline Minimum & 0.0 & 0.0 & \\
\hline Median & 0.0 & 2.0 & \\
\hline Maximum & 9.0 & 7.0 & \\
\hline \multicolumn{4}{|l|}{ Medical History, n (\%) } \\
\hline Vaso-occlusive crisis & $25(61.0)$ & $12(57.1)$ & \\
\hline Acute chest syndrome & $9(22.0)$ & $2(9.5)$ & \\
\hline Hepatic sequestration & 0 & $1(4.8)$ & \\
\hline Splenectomy & $7(17.1)$ & $5(23.8)$ & \\
\hline Systemic hypertension & $11(26.8)$ & $2(9.5)$ & \\
\hline Pulmonary hypertension & $7(17.1)$ & $2(9.5)$ & \\
\hline Renal failure & $1(2.4)$ & 0 & \\
\hline Hepatic disease $^{\dagger}$ & $1(2.4)$ & 0 & \\
\hline Renal insufficiency & 0 & $1(4.8)$ & \\
\hline \multicolumn{4}{|l|}{ Laboratory Values, mean (SD) } \\
\hline Hemoglobin (g/L) & $10.4(1.8)$ & $9.8(2.0)$ & 0.297 \\
\hline White Blood Cells (x109/L) & $8.2(3.4)$ & $8.3(2.7)$ & 0.929 \\
\hline Platelets (x109/L) & $310.6(180.4)$ & $340.5(117.5)$ & 0.561 \\
\hline
\end{tabular}

*1 patient was found not to have SCD but to have only $\beta$-thalassemia trait. ${ }^{\dagger}$ Other than sequestration.

discontinuation. The proportion of SAEs and all TEAEs was similar between the groups. Most SAEs were pain events requiring medical intervention. The rate of study drug discontinuation was similar between treatment groups. No patients discontinued the study due to an AE. There were 2 AEs that led to study drug discontinuation in the prasugrel arm, but neither of these events was hemorrhagic or deemed by the investigator to be related to the study drug. The events were pain not otherwise specified and bronchitis. There were no clinically significant differences in the routine laboratory values (complete blood count, chemistry panels, and liver function tests) with serial follow-up (data not shown).

The efficacy endpoints in this study were the rate and intensity of pain as recorded by daily self-administered pain diaries. The mean completion percentage for diaries (number of entries/total possible days) was $95.7 \%$ for the prasugrel arm and $92.6 \%$ for the placebo arm. There were numerical decreases in proportion of days with pain and pain intensity in the prasugrel treated patients: a $21 \%$ relative reduction (least-squares [LS] mean: $42.2 \%$ vs. $53.5 \%$ ) in pain rate and a relative $25 \%$ reduction (LS mean: 1.8 vs. 2.4) in pain intensity. However, these differences did not reach statistical significance. An alternative analysis showed that a greater proportion of patients in the prasugrel arm (50\%) reported no pain throughout the study $(27.5 \%$ of patients with a percentage of days with pain $=0$ ) or infrequent pain (22.5\% of patients with $\leq 25 \%$ of days with pain) versus $22.3 \%$ of placebo-treated patients having either no pain $5.6 \%$ of patients with a percentage of days with 
Table 2 Summary of adverse events

\begin{tabular}{lcc}
\hline & $\begin{array}{c}\text { Prasugrel } \\
(\mathbf{n}=\mathbf{4 1 )}\end{array}$ & $\begin{array}{c}\text { Placebo } \\
(\mathbf{n}=\mathbf{1 9})\end{array}$ \\
\hline $\begin{array}{l}\text { Any hemorrhagic event, } \mathbf{n}(\%) \\
\text { Required medical attention }\end{array}$ & 0 & 0 \\
Treatment-emergent & $8(19.5)$ & $1(5.3)$ \\
Possibly related to study drug & $6(14.6)$ & $1(5.3)$ \\
Any non-hemorrhagic event, $\mathbf{n}(\%)$ & & \\
Serious & $8(19.5)$ & $4(21.1)$ \\
Study drug discontinuation & $2(4.9)$ & 0 \\
Treatment-emergent & $31(75.6)$ & $17(89.5)$ \\
Possibly related to study drug & $2(4.9)$ & 0 \\
Any event, $\mathbf{n}$ (\%) & & \\
Serious & $8(19.5)$ & $4(21.1)$ \\
Study drug discontinuation & $2(4.9)$ & 0 \\
Treatment emergent & $34(82.9)$ & $17(89.5)$ \\
Possibly related to study drug & $8(19.5)$ & $1(5.3)$ \\
\hline
\end{tabular}

pain $=0)$ or infrequent pain $(16.7 \%$ of patients with $\leq$ $25 \%$ of days with pain) (Figure $3 \mathrm{~A})$. Nine of 40 (22.5\%) of prasugrel patients had painful episodes during the study period versus $7 / 19$ (36.8\%) in the placebo group. Again, this was not statistically different.

The point-of-care VerifyNow ${ }^{\circledR}$ P2Y12 device and VASP PRI were used to assess prasugrel's pharmacodynamic effect on platelets. Figure 4 shows that by both measures, $5 \mathrm{mg}$ prasugrel resulted in statistically significant greater platelet inhibition compared to placebo $(P<.001)$ at both day 10 and day 30 . About $20 \%$ of patients had $<20 \%$ inhibition, which is consistent with the rate seen with the 5-mg maintenance dose in previous studies [24-26]. Because the proportion of patients did not fulfill the pre-specified criterion for escalation, the dose remained at $5 \mathrm{mg}$ for the duration of the study.
Numerous studies have shown that platelets are activated in SCD patients $[10,12]$ and that markers of platelet activation are associated with complications $[13,14]$. The effects of prasugrel on platelet activation were measured with both cellular (platelet P-selectin) and soluble biomarkers (TXB2, soluble CD40L, and soluble P-selectin). There were statistically significant decreases in platelet P-selectin and soluble P-selectin at both days 10 and 30 in the prasugrel arm compared to placebo. The decreases also reached statistical significance for TXB2 at day 10 and for soluble CD40L at day 30 in the prasugrel arm compared to placebo (Figure 5). Therefore, prasugrel was shown not only to inhibit platelet function, but also to decrease platelet activation in patients with SCD.

\section{Discussion}

In this randomized Phase 2 study of prasugrel in adult patients with various genotypes of SCD in steady state, 30 days of treatment was not associated with hemorrhagic events that required immediate medical attention. As expected for a platelet function inhibitor, there were more hemorrhagic AEs with prasugrel; however, the majority was mild and none resulted in serious adverse events or adverse events leading to study drug or study discontinuation. However, it bears emphasis that exclusion criteria were conservative, enrolled subjects were at low risk for hemorrhage and this pilot study was of short duration.

Evidence of platelet inhibition was seen amongst prasugrel-treated patients with SCD. Prasugrel treatment resulted in approximately $33 \%$ to $40 \%$ platelet inhibition as measured by both VerifyNow ${ }^{\circledR}$ P2Y12 and VASP, suggesting that patients with SCD achieved a substantial pharmacodynamic response with prasugrel treatment similar to that seen in previous studies in patients with cardiovascular disease. Despite this effect, it was still

Table 3 Hemorrhagic adverse events

\begin{tabular}{|c|c|c|c|c|}
\hline Treatment & Patient & Preferred term & Severity & Possibly related to study drug \\
\hline \multirow[t]{3}{*}{ Placebo } & 1 & Hematochezia & Mild & \\
\hline & & Gingival bleeding & Moderate & Yes \\
\hline & & Hemorrhoids & Mild & \\
\hline \multirow[t]{9}{*}{ Prasugrel } & 2 & Menorrhagia & Moderate & Yes \\
\hline & 3 & Menorrhagia & Mild & Yes \\
\hline & & Ecchymosis & Mild & \\
\hline & 4 & Epistaxis & Mild & Yes \\
\hline & 5 & Contusion & Mild & Yes \\
\hline & 6 & Epistaxis & Mild & \\
\hline & 7 & Gingival bleeding & Mild & Yes \\
\hline & 8 & Gingival bleeding & Mild & Yes \\
\hline & 9 & Hematochezia & Mild & \\
\hline
\end{tabular}



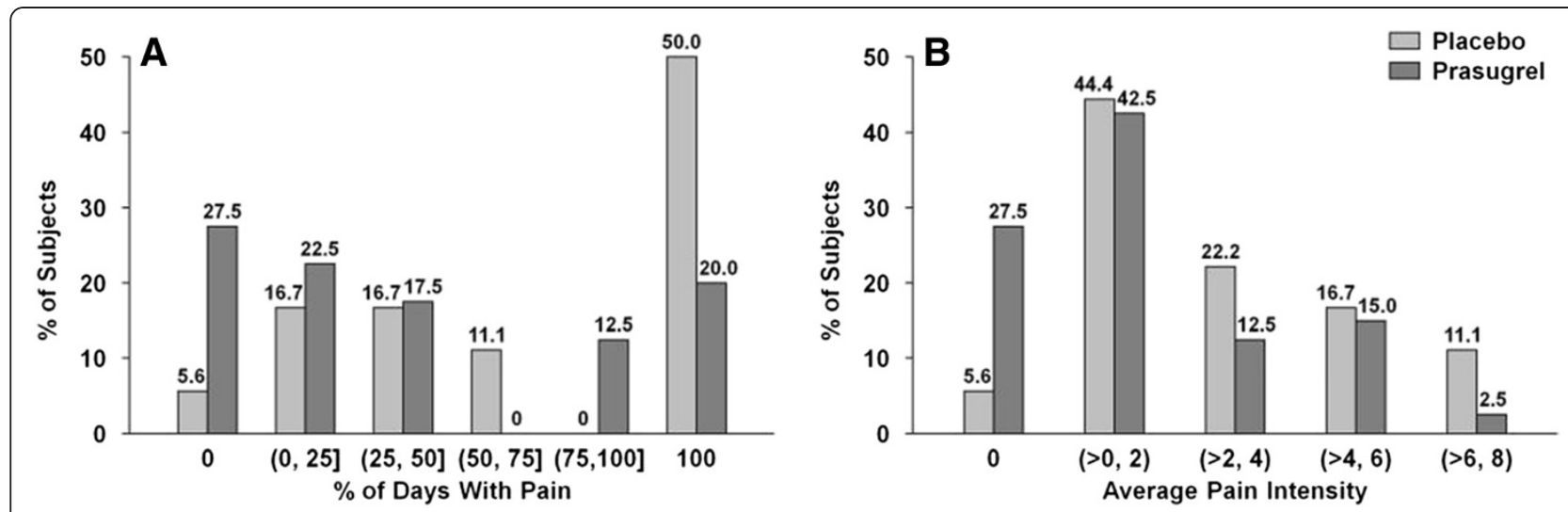

Figure 3 Patient-reported days with pain and pain intensity. A. Proportion of patients reporting pain on $0,>0$ to $25,>25$ to $50,>50$ to $75,>75$ to $<100$, or $100 \%$ of study days. B. Proportion of patients with average pain intensity of $0,>0$ to $2,>2$ to $4,>4$ to 6 , or $>6$ to 8 . Prasugrel $=$ black bars; placebo $=$ grey bars.

possible that the marked hemostatic and inflammatory milieu in SCD patients might have overcome potential inhibition of platelet activation by prasugrel via other platelet activation pathways, such as thrombin generation. However, the decreased levels in cellular and soluble markers of platelet activation observed in the prasugrel-treated group show this agent is able to overcome this platelet pro-stimulatory environment and attenuate in vivo platelet activation in SCD without producing serious bleeding.

Perhaps more provocative is the suggestion that prasugrel may be efficacious in reducing pain. The traditional endpoint for therapeutic efficacy in SCD is the occurrence of acute painful episodes (pain that requires an unscheduled visit to a medical facility for treatment). However, paradigm shifting work by Smith et al. [27,28]. has shown that chronic pain is common in patients with SCD. Therefore, reductions in pain rate (the proportion of days with pain) and pain intensity over time are important endpoints for SCD therapy to assess efficacy in treating the different types of pain problems experienced in this patient population. A numerical decrease in pain rate and intensity compared with placebo, as reported by daily patient diaries, was seen in this study. There was also a numerical decrease in acute pain episodes compared with placebo. Neither of these decreases reached statistical significance, and this pilot study was not designed to provide definitive conclusions regarding pain. However, the number of patients who reported days with zero pain was different. This latter finding was intriguing, as it is a measurement of reduction of both acute pain episodes and chronic pain and was seen after only 30 days of treatment, a more rapid time to response than seen with hydroxyurea [1].

It is important to compare and contrast the results of this study to the other published studies of platelet

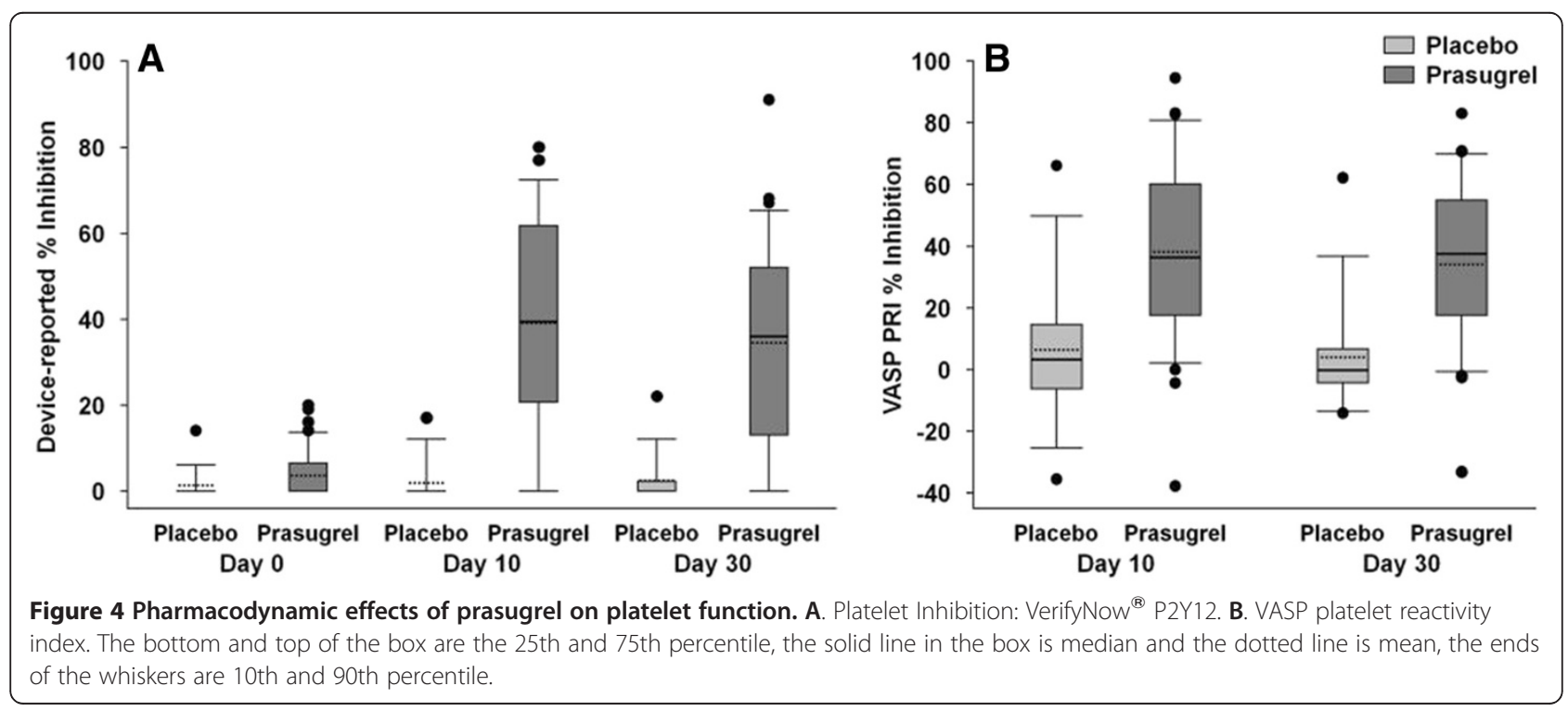



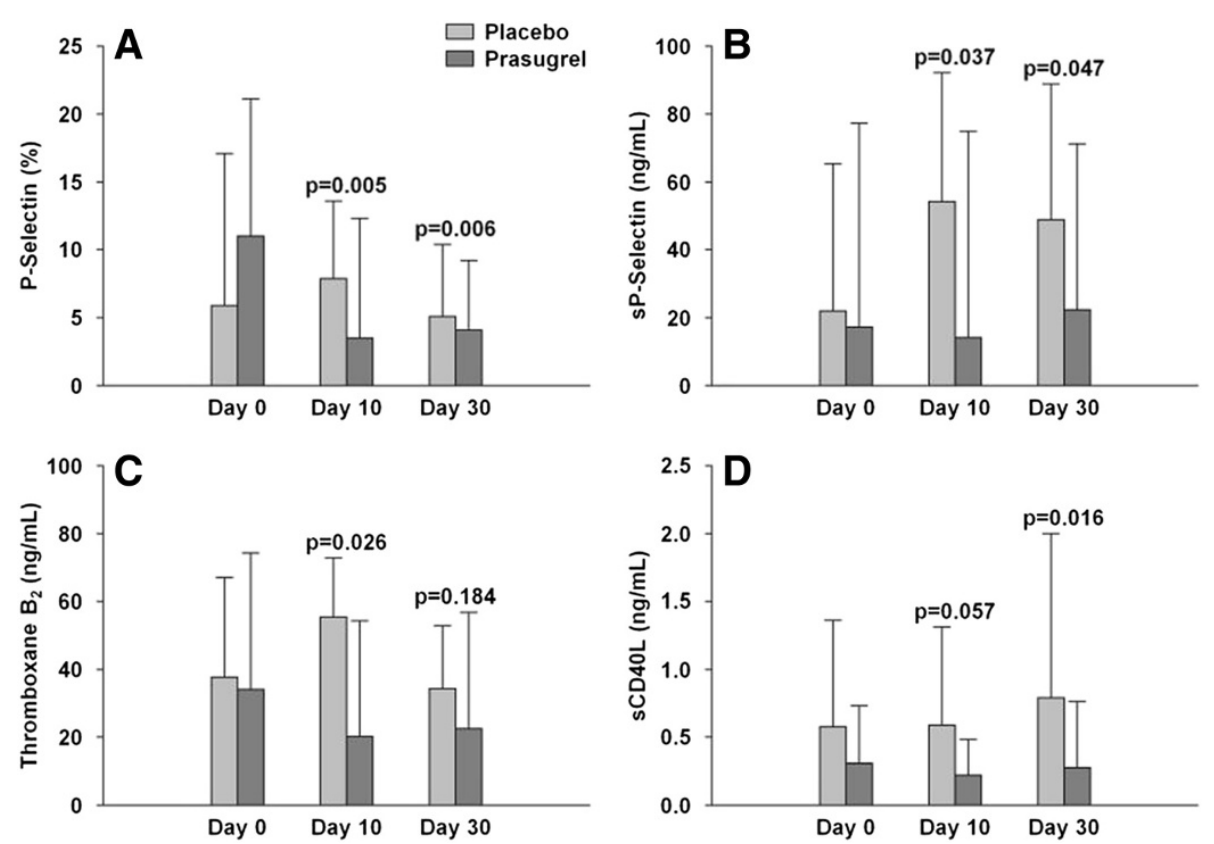

Figure 5 Effect of prasugrel vs. placebo on biomarkers of disease-related platelet activation. A. Percent of platelets positive for platelet surface P-selectin, B. Plasma soluble P-selectin, C. Serum TXB2, D. plasma soluble CD40L. Prasugrel = black bars; placebo=grey bars. Results are mean \pm SD.

inhibitors in SCD. Osamo et al. examined the effect of aspirin on red cell survival in patients with SCD [15]. Fifty patients aged $11-20$ years with HbSS were assigned to $1200 \mathrm{mg}$ daily of soluble aspirin in divided doses for 6 weeks; another 50 were assigned to usual care only. Hemoglobin and oxygen saturation levels increased in the treated group, and red cell survival increased in the 3 patients in whom it was studied. They also demonstrated a shift in the electrophoretic mobility of $\mathrm{HbS}$ in the aspirintreated patients indicating chemical modification of $\mathrm{HbS}$. Pain was not formally assessed in this study, and no serious hemorrhagic events were reported.

Greenberg et al. studied somewhat lower doses of aspirin $(3-6 \mathrm{mg} / \mathrm{kg}$ ) for 21 months in 49 children with $\mathrm{HbSS}, \mathrm{HbSC}$, or HbSO-Arab in a double-blind crossover study to prevent acute pain crises [29]. Ninety-four patients were originally enrolled but analysis was per protocol: only 49 that were determined to be at least $50 \%$ adherent with the study drug were included in the analysis cohort. There was no difference in the number of painful episodes, number of total days in pain, duration of pain crisis, or pain severity during crisis between the aspirin- and placebo-treated periods. Interestingly, there was a marked decrease in the number of pain crises after the first 6 months on-study, irrespective of the treatment assignment.

Chaplin and colleagues added the phosphodiesterase inhibitor dipyridamole to aspirin as prophylaxis for acute pain crisis [30]. This study included only 3 patients treated with aspirin $650 \mathrm{mg} \mathrm{PO}$ and dipyridamole $50 \mathrm{mg}$ PO both twice daily and compared the frequency and severity of pain for the 2 years on therapy to the 2 years not on therapy. The severity of pain appeared to be less while on therapy, and the total number of hospitalizations for pain decreased as well.

Previous studies have tested the effect of the first generation thienopyridine ticlopidine in SCD patients. Semple and colleagues assessed platelet survival in 9 patients with SCD using radiolabeled platelets and platelet activation by measuring plasma levels of platelet release products [17]. Patients were randomized to placebo or ticlopidine $250 \mathrm{mg}$ PO twice daily for 28 days. Ticlopidine did not prolong platelet survival but did decrease markers of platelet activation, as was seen in our study. They noted an approximate $40 \%$ reduction in collagen and ADPinduced maximal platelet aggregation. The inhibition of platelet function and relative reduction in markers of platelet activation is similar, in degree, to that seen in the present study using different assays. One patient had a painful episode while on active drug, but clearly this study was not powered to determine a difference in pain. Adverse events were not reported.

Cabannes et al. studied the efficacy of ticlopidine in the prevention of acute pain crisis in 140 patients in Africa [6]. Patients were randomized 1:1 between ticlopidine $500 \mathrm{mg}$ to $750 \mathrm{mg}$ daily (dependent on body weight) or placebo for 6 months of therapy. Although the precise definitions of crisis, crisis duration, and crisis 
severity cannot be discerned from the report, all three of these parameters were statistically significantly decreased in the ticlopidine arm compared with the placebo arm. No platelet activation or survival markers were assessed.

Our study showed similar effects on platelet activation to those demonstrated with ticlopidine, confirming its platelet suppressive effect in this population of patients without provoking clinically serious bleeding. It is important to note that the current Phase 2 study was not powered to find a difference in acute painful episodes. Nonetheless, there was a trend toward decreased number of days with pain. Treatment with prasugrel compared with placebo was associated with numerical decreases in pain rate and pain intensity, as reported in daily patient diaries, and a numerical decrease in pain events related to SCD that required medical attention, as assessed by the study investigator. In adjusted analyses, prasugrel was associated with a $21 \%$ relative reduction in the percentage of days with pain and a $25 \%$ relative reduction in pain intensity compared with placebo.

Although it is clear from the patient pain diaries that the majority of patients had pain at some point during the study and many of the patients randomized to placebo experienced chronic pain, relatively few patients sought medical attention for their pain (27\%). From the case report forms, $22.5 \%$ of prasugrel-treated patients sought medical attention for a pain event versus $36.8 \%$ of placebo-treated patients. These provide a signal that prasugrel may be efficacious in reducing the frequency and severity of VOC in patients with SCD.

\section{Conclusion}

In summary, the results of this randomized, doubleblind, Phase 2 study showed that prasugrel inhibited platelet function, decreased biomarkers of platelet activation, and showed a trend toward decreased pain by several measures without serious hemorrhagic adverse events. However, these results must be interpreted with caution, as measures of pain were not primary outcomes and the duration of this study (30 days) was short. A larger study with a sample size appropriately powered to determine effects on clinically relevant endpoints and provide longer safety analysis is needed to confirm and extend these findings.

\section{Competing interests}

CZ, LEH, KJW, JSR, and JAJ, Eli Lilly and Company Employment and Equity Ownership. TW, LK, LEM, JJS, Daiichi Sankyo Co., Ltd. and Eli Lilly and Company Research Funding. CEN, Daiichi Sankyo, Inc. Employment and Equity Ownership. AK, Research funding from Novartis, Celgene, Hemaquest, Daiichi Sankyo Co., Ltd., and Eli Lilly and Company. KIA, Adventrx Consultancy, Daiichi Sankyo Co., Ltd. and Eli Lilly and Company Research Funding. ALF, PhD Research funding: Daiichi Sankyo Co., Ltd. and Eli Lilly and Company, GLSynthesis, Glycomimetics; Consultant: Eli Lilly and Company. CLK, DS, EN, no financial relationship(s) to disclose.

\section{Authors' contributions}

TW, participated in the design of the study, chaired the study, performed the research, and wrote the manuscript. ALF III performed research (assayed selected biomarkers); contributed to manuscript EN, performed research (contributed patients to the study), contributed to manuscript DS, FRCPC, MSc, performed research (contributed patients to the study), contributed to manuscript. LK, performed research (contributed patients to the study), contributed to manuscript. AK, performed research (contributed patients to the study), contributed to manuscript KA, performed research (contributed patients to the study), contributed to manuscript. CZ MS, participated in the design of the study, analysis and interpretation of the data, contributed to manuscript and performed the statistical analyses. LEH, participated in the design of the study, analysis and interpretation of the data, contributed to manuscript. CEN, participated in the design of the study, contributed to manuscript. JAJ, participated in the design of the study, analysis and interpretation of the data, contributed to manuscript. KJW, participated in the design of the study, analysis and interpretation of the data, contributed to manuscript. JSR, participated in the design of the study, contributed to manuscript. CLK, performed research (contributed patients to the study), assisted with revisions of the manuscript. LEM, Performed research (contributed patients to the study), contributed to manuscript. JJS Performed research (contributed patients to the study), contributed to manuscript. All authors read and approved the final manuscript.

\section{Acknowledgements}

This study was funded by Daiichi Sankyo Co., Ltd. and Eli Lilly and Company. Maryann Weller, PharmaNet/i3, formatting and editing.

\section{Study team}

Carolyn Bigelow, University of Mississippi Health, Jackson, MS, USA Loray Blair-Britt, Century Clinical Research Inc., Daytona Beach, FL, USA Archie Hearne, Preferred Research Partners LLC, Little Rock, AK, USA Marvin Kalafer, Clinical Trial Center, Jenkintown, PA, USA

Stella Kamanda, Little Rock Cancer Clinic, Little Rock, AK, USA Derek Lewis, North Little Rock Primary Care and Diagnostic Clinic, Little Rock, AK, USA

Floyd Luckett, Trialogic Research at Immediate Medical Care, Houston, TX, USA

Michele Sewell, IALUM Clinical Research Center, Stone Mountain, GA, USA Amy Shapiro, Indiana Hemophilia and Thrombosis Center, Indianapolis, IN USA

Mitchel Scott Touger, Innovative Clinical Trials, Birmingham, AL, USA

Ted Wun, MD, University of California, Sacramento, CA

Andrew L. Frelinger III, Ph.D. Children's Hospital Boston, Harvard Medical

School, Boston, MA

Denis Soulieres, MD, FRCPC, MSC, CHUM-Notre-Dame, Montreal, QC, Canada Lakshmanan Krishnamurti, MD, Children's Hospital of Pittsburgh, Pittsburgh, PA

Abdullah Kutlar, MD, Medical College of Georgia, Augusta, GA

Kenneth Ataga, MD, University of North Carolina at Chapel Hill, Chapel Hill, NC

Charles L. Knupp, M.D., Hematology/Oncology, ECU-Brody School of Medicine, Greenville, NC, USA

Lillian E. McMahon, M.D., Hematology/Oncology, Boston Medical Center, Boston, MA, USA

John J. Strouse, M.D., Ph.D., Johns Hopkins University School of Medicine, Baltimore, MD, USA

\section{Author details}

'University of California, Davis Cancer Center, 4501 X St., Ste. 3016, Sacramento, CA 95817, USA. ${ }^{2}$ CHUM-Notre-Dame, Montreal, QC, Canada. ${ }^{3}$ Children's Hospital Boston, Harvard Medical School, Boston, MA, USA. ${ }^{4}$ Children's Hospital of Pittsburgh, Pittsburgh, PA, USA. ${ }^{5}$ University of Pittsburg Medical Center, Pittsburg, PA, USA. ${ }^{6}$ Medical College of Georgia, Augusta, GA, USA. 'University of North Carolina at Chapel Hill, Chapel Hill, NC, USA. ${ }^{8}$ ECU-Brody School of Medicine, Greenville, NC, USA. 'Boston Medical Center, Boston, MA, USA. ${ }^{10}$ The Johns Hopkins University School of Medicine, Baltimore, MD, USA. ${ }^{11}$ Eli Lilly and Company, Indianapolis, IN, USA.

${ }^{12}$ Daiichi Sankyo, Inc, Edison, NJ, USA. 
Received: 19 December 2012 Accepted: 5 February 2013

Published: 17 February 2013

\section{References}

1. Steinberg MH: Management of sickle cell disease. N Engl J Med 1999, 340:1021-1030.

2. Hebbel RP, Boogaerts MA, Eaton JW, Steinberg MH: Erythrocyte adherence to endothelium in sickle-cell anemia. A possible determinant of disease severity. N Engl J Med 1980, 302:992-995.

3. Wun T, Cordoba M, Rangaswami A, Cheung AW, Paglieroni T: Activated monocytes and platelet-monocyte aggregates in patients with sickle cell disease. Clin Lab Haematol 2002, 24:81-88.

4. Lum AF, Wun T, Staunton D, Simon SI: Inflammatory potential of neutrophils detected in sickle cell disease. Am J Hematol 2004, 76:126-133.

5. Haynes J Jr, Obiako B: Activated polymorphonuclear cells increase sickle red blood cell retention in lung: role of phospholipids. Am J Physiol Heart Circ Physiol 2002, 282:H122-H130.

6. Frenette PS: Sickle cell vasoocclusion: heterotypic, multicellular aggregations driven by leukocyte adhesion. Microcirculation 2004 11:167-177.

7. Ataga Kl, Moore CG, Hillery CA, Jones S, Whinna HC, Strayhorn D, Sohier C, Hinderliter A, Parise LV, Orringer EP: Coagulation activation and inflammation in sickle cell disease-associated pulmonary hypertension. Haematologica 2008, 93:20-26.

8. Ataga Kl, Key NS: Hypercoagulability in sickle cell disease: new approaches to an old problem. Hematology Am Soc Hematol Educ Program 2007, 2007:91-96.

9. Ataga Kl, Cappellini MD, Rachmilewitz EA: Beta-thalassaemia and sickle cell anaemia as paradigms of hypercoagulability. Br J Haematol 2007, 139:3-13.

10. Wun T, Paglieroni T, Rangaswami A, Franklin PH, Welborn J, Cheung A, Tablin F: Platelet activation in patients with sickle cell disease. Br J Haematol 1998, 100:741-749.

11. Ataga Kl, Orringer EP: Hypercoagulability in sickle cell disease: a curious paradox. Am J Med 2003, 115:721-728.

12. Tomer A, Harker LA, Kasey S, Eckman JR: Thrombogenesis in sickle cell disease. J Lab Clin Med 2001, 137:398-407.

13. Villagra J, Shiva S, Hunter LA, Machado RF, Gladwin MT, Kato GJ: Platelet activation in patients with sickle disease, hemolysis-associated pulmonary hypertension, and nitric oxide scavenging by cell-free hemoglobin. Blood 2007, 110:2166-2172.

14. Ataga Kl, Brittain JE, Desai P, May R, Jones S, Delaney J, Strayhorn D, Hinderliter A, Key NS: Association of coagulation activation with clinical complications in sickle cell disease. PLOS One 2012, 7:e29786.

15. Osamo NO, Photiades DP, Famodu AA: Therapeutic effect of aspirin in sickle cell anaemia. Acta Haematol 1981, 66:102-107.

16. Cabannes R, Lonsdorfer J, Castaigne JP, Ondo A, Plassard A, Zohoun I: Clinical and biological double-blind-study of ticlopidine in preventive treatment of sickle-cell disease crises. Agents Actions Supp/ 1984, 15:199-212.

17. Semple MJ, Al-Hasani SF, Kioy P, Savidge GF: A double-blind trial of ticlopidine in sickle cell disease. Thromb Haemost 1984, 51:303-306.

18. Blann AD, Mohan JS, Bareford D, Lip GY: Soluble P-selectin and vascular endothelial growth factor in steady state sickle cell disease: relationship to genotype. J Thromb Thrombolysis 2008, 25:185-189.

19. Browne PV, Mosher DF, Steinberg MH, Hebbel RP: Disturbance of plasma and platelet thrombospondin levels in sickle cell disease. Am J Hematol 1996, 51:296-301.

20. Varenhorst C, James S, Erlinge D, Braun OO, Brandt JT, Winters KJ, Jakubowski JA, Olofsson S, Wallentin L, Siegbahn A: Assessment of P2Y(12) inhibition with the point-of-care device VerifyNow P2Y12 in patients treated with prasugrel or clopidogrel coadministered with aspirin. Am Heart J 2009, 157:562-569.

21. Jakubowski JA, Payne CD, Li YG, Brandt JT, Small DS, Farid NA, Salazar DE, Winters KJ: The use of the VerifyNow P2Y12 point-of-care device to monitor platelet function across a range of $\mathrm{P} 2 \mathrm{Y} 12$ inhibition levels following prasugrel and clopidogrel administration. Thromb Haemost 2008, 99:409-415.

22. Michelson AD, Barnard MR, Krueger LA, Valeri CR, Furman MI: Circulating monocyte-platelet aggregates are a more sensitive marker of in vivo platelet activation than platelet surface P-selectin: studies in baboons, human coronary intervention, and human acute myocardial infarction. Circulation 2001, 104:1533-1537.

23. Frelinger AL III, Michelson AD, Wiviott SD, Trenk D, Neumann FJ, Miller DL, Jakubowski JA, Costigan TM, McCabe CH, Antman EM, Braunwald E: Intrinsic platelet reactivity before $\mathrm{P} 2 \mathrm{Y} 12$ blockade contributes to residual platelet reactivity despite high-level P2Y12 blockade by prasugrel or high-dose clopidogrel. Results from PRINCIPLE-TIMI 44. Thromb Haemost 2011, 106:219-226.

24. Jakubowski JA, Payne CD, Weerakkody GJ, Brandt JT, Farid NA, Li YG, Naganuma H, Lachno DR, Winters KJ: Dose-dependent inhibition of human platelet aggregation by prasugrel and its interaction with aspirin in healthy subjects. J Cardiovasc Pharmacol 2007, 49:167-173.

25. Jakubowski JA, Winters KJ, Naganuma H, Wallentin L: Prasugrel: a novel thienopyridine antiplatelet agent. A review of preclinical and clinical studies and the mechanistic basis for its distinct antiplatelet profile. Cardiovasc Drug Rev 2007, 25:357-374.

26. Jernberg T, Payne CD, Winters KJ, Darstein C, Brandt JT, Jakubowski JA, Naganuma $H$, Siegbahn A, Wallentin L: Prasugrel achieves greater inhibition of platelet aggregation and a lower rate of non-responders compared with clopidogrel in aspirin-treated patients with stable coronary artery disease. Eur Heart J 2006, 27:1166-1173.

27. Smith WR, Bovbjerg VE, Penberthy LT, McClish DK, Levenson JL, Roberts JD, Gil K, Roseff SD, Aisiku IP: Understanding pain and improving management of sickle cell disease: the PiSCES study. J Natl Med Assoc 2005, 97:183-193.

28. Smith WR, Penberthy LT, Bovbjerg VE, McClish DK, Roberts JD, Dahman B, Aisiku IP, Levenson JL, Roseff SD: Daily assessment of pain in adults with sickle cell disease. Ann Intern Med 2008, 148:94-101.

29. Greenberg J, Ohene-Frempong K, Halus J, Way C, Schwartz E: Trial of low doses of aspirin as prophylaxis in sickle cell disease. J Pediatr 1983, 102:781-784.

30. Chaplin H Jr, Alkjaersig N, Fletcher AP, Michael JM, Joist JH: Aspirindipyridamole prophylaxis of sickle cell disease pain crises. Thromb Haemost 1980, 43:218-221.

doi:10.1186/1756-8722-6-17

Cite this article as: Wun et al:: A double-blind, randomized, multicenter phase 2 study of prasugrel versus placebo in adult patients with sickle cell disease. Journal of Hematology \& Oncology 2013 6:17.

\section{Submit your next manuscript to BioMed Central and take full advantage of:}

- Convenient online submission

- Thorough peer review

- No space constraints or color figure charges

- Immediate publication on acceptance

- Inclusion in PubMed, CAS, Scopus and Google Scholar

- Research which is freely available for redistribution 